\title{
Radio Resource Management in Heterogeneous Deployments: a System Level Perspective
}

\author{
Thomas Wirth, Johannes Dommel, Kai Börner, Lars Thiele, Thomas Haustein \\ Fraunhofer Institute for Telecommunications \\ Heinrich Hertz Institute \\ Einsteinufer 37, 10587 Berlin, Germany \\ thomas.wirth@hhi.fraunhofer.de
}

\begin{abstract}
New multimedia services such as HTTP live video streaming [1] demand for higher capacity, especially in mobile networks. In fact, about $50 \%$ of data in mobile networks is currently video traffic, and this number is expected to increase to $70-80 \%$ by 2015 [2]. To enhance the capacity in cellular networks even further, several study items were defined [3] to target peak data rates of up to $1 \mathrm{Gbit} / \mathrm{s}$ in the downlink and $500 \mathrm{Mbit} / \mathrm{s}$ in the uplink. The different techniques are currently being discussed within the 3GPP for LTEs evolution called LTE-Advanced (LTE-A). A promising approach to enhance the capacity in cellular access networks even further is to create smaller cells [4]. This represents a shift in paradigm from Macro cells to a more heterogeneous ecosystem consisting of a combination of different cell sizes and transmission powers and thus coverage, referred to as Macro-, Pico-, and Femto-cells.
\end{abstract}

The key idea behind heterogeneous networks (HetNet) is that the frequency reuse factor (FRS) is set to 1 , which means that all heterogeneous cells operate in the same frequency range, but can be controlled and optimized in frequency domain together. With FRS 1, cell capacity is strongly interference limited and interference management (IM) techniques are required at both base station (eNB) and user equipment (UE) to reach the target performance. Newly defined protocols between HetNet entities allow decentralized, feedback-based radio resource management (RRM) which can help to mitigate interference and enhance HetNet performance on a cellular level. The performance is measured by key performance indicators (KPIs) [5] with performance criteria tailored to HetNet deployments. Aim of this paper is to evaluate RRM techniques which focus on a distributed precoding concept on system-level taking into account realistic antenna models optained by $3 \mathrm{D}$ antenna measurements.

System Model: We consider a HetNet system with a set of Macro cells and 1 or 2 associated Pico cells per Macro. Considering effect on the wireless channel, e.g. path loss, shadow fading, 3D antenna models, the received downlink signal $\mathbf{y}_{m}$ at user $m$ and is given by

$$
\mathbf{y}_{m}=\underbrace{\mathbf{H}_{i, m} \mathbf{b}_{i, m}}_{\overline{\mathbf{h}}_{m}} x_{i, m}+\underbrace{\sum_{j \in \mathcal{M} \backslash\{m\}} \mathbf{H}_{i, m} \mathbf{b}_{i, j} x_{i, j}}_{\zeta_{m}}+\mathbf{z}_{m}
$$

The desired data stream $x_{i, m}$ transmitted to the $m^{\text {th }}$ user from the $i^{\text {th }}$ cell is distorted by the intra- and inter-cell interference plus noise aggregated in $\zeta_{m}$ and $\mathbf{z}_{m}$, respectively. $\mathbf{H}_{i, m}$ spans the $N_{R} \times N_{T}$ channel matrix for user $m$ formed by the $i^{t h}$ cell. The $N_{T} \times N_{T}$ precoding matrix $\mathbf{B}_{i}=\left[\mathbf{b}_{i, 1} \cdots \mathbf{b}_{i, M}\right]$ includes the power allocation $p_{i}$ and contains the precoders $\mathbf{b}_{i, m}$ designed for each user. The proposed precoding matrix treats the Macro and associated Picos as a virtual antenna array and forms a combined virtual precoding matrix.

The precoder is defined by the codebooks $\mathbf{B}_{i}$ with Macro power weights $\gamma$, and Pico power weights $\alpha$ and $\beta$, which fulfill the corresponding per-antenna power constraints of the transmit antennas.
The precoding codebooks for a Macro eNB with 2 transmit antenna and 2 Pico eNBs with a single antenna can be written as

$$
\begin{aligned}
& \mathbf{B}_{i}:=\underbrace{\left[\begin{array}{cccc}
\gamma & \gamma & 0 & 0 \\
\gamma & -\gamma & 0 & 0 \\
0 & 0 & 0 & 0 \\
0 & 0 & 0 & 0
\end{array}\right]}_{\mathbf{a})} \underbrace{\left[\begin{array}{cccc}
\gamma & \gamma & 0 & 0 \\
\gamma & -\gamma & 0 & 0 \\
0 & 0 & \alpha & 0 \\
0 & 0 & 0 & 0
\end{array}\right]}_{\text {b) }} \\
& \underbrace{\left[\begin{array}{cccc}
\gamma & \gamma & 0 & 0 \\
\gamma & -\gamma & 0 & 0 \\
0 & 0 & 0 & 0 \\
0 & 0 & 0 & \beta
\end{array}\right]}_{\mathbf{c})} \underbrace{\left[\begin{array}{cccc}
\gamma & \gamma & 0 & 0 \\
\gamma & -\gamma & 0 & 0 \\
0 & 0 & \alpha & 0 \\
0 & 0 & 0 & \beta
\end{array}\right]}_{\mathbf{d})}
\end{aligned}
$$

Here, the colums of matrix $B$ represent the number of transmit antennas and the rows the number of receive antennas. The precoder $a$ ) implies that associated Picos are switched off and data is transmitted only via the Macro cell. If precoding vector $b$ ) and $c$ ) are used, data is transmitted either using the Macro and one or two Pico cells. In the case precoding vector $d$ ) is used, data is transmitted by the Macro sector as well as both Pico cells. This system can be enhanced in case the number of antennas is increased at the Macro and/or Pico, respectively.

Radio Resource Management Concept: The RRM is divided into radio resource assignment (RRA) and long-term radio resource management. Both include interference management as well as mobility and load management. The distributed RRM entities within the HetNet scenario operate in a master-slave mode where the Macro eNB operates as a master, and the associated Pico eNB run in slave mode. Macro- and Pico cells are interconnected via the X2 interface which is used to distribute scheduling maps and precoding matrices $\mathbf{B}_{i}$. The mobility management can push UEs into the Pico cell or handover UEs into the Macro sector depending on the link budget, traffic load to a given UE as well as a handover margin to avoid handover hysteresis. Macro sector and Pico cells cooperate during RRA in time, frequency and spatial domains. By applying precoding matrices from Eq. 2 on a subchannel basis, Pico spectrum can be switched on and off in parts of the frequency domain. Here, subchannel refers to a group of subcarriers typically consisting of 4-6 physical resource blocks (PRBs). Finally, scheduling maps and proding matrices can be updated on different time scales. Depending on the load and mobility and the capacity of the interfaces between nodes within the HetNet scenario, the feedback updated frequency can be either on a basis of several milliseconds up to several seconds and can be on a regular time frame or base on a trigger from the master eNB.

System Level Simulation Environment: The RRM algorithms are analyzed by system-level simulations. The main parameters sets considered in the simulation are summarized in Table I. For realistic antenna modelling, we use a 3D antenna model which is described 

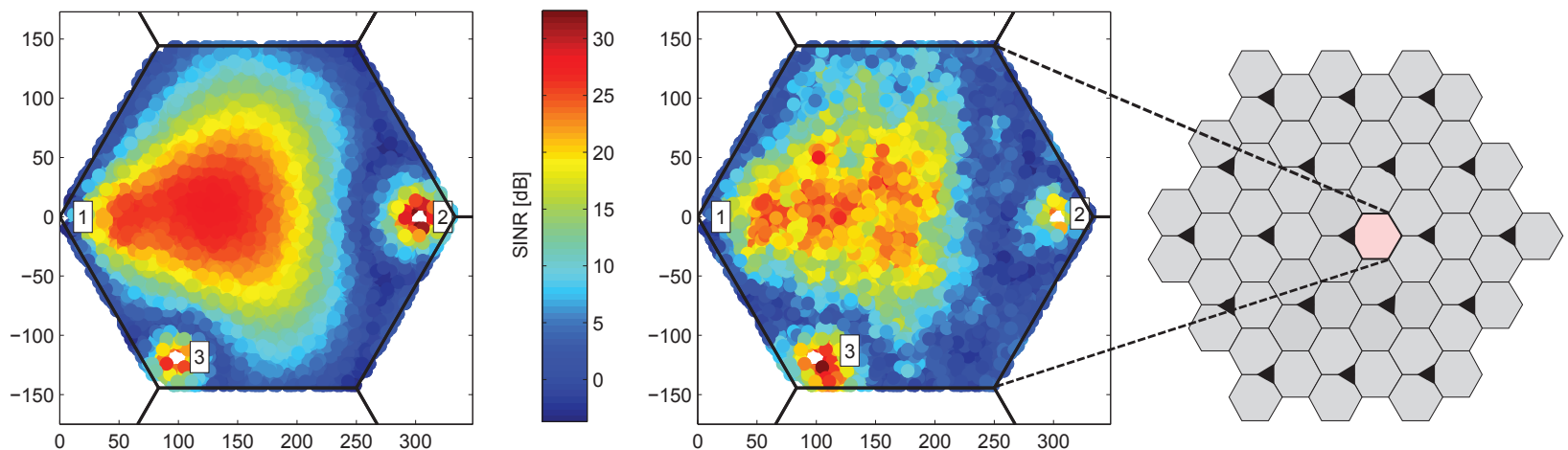

Fig. 1. The HetNet simulation scenario shows the cell-of-interest which is 1 Macro eNB sector surrounded by 57 Macro eNBs sectors. The cell-of-interest is configured with 2 Pico eNBs located at the far right and left bottom of the Macro sector. Position 1 represents the Macro eNB sector, positions 2 and 3 the eNBs Pico cells. The color code represents the SINR [dB] of the scenario, on the (left) side without shadow fading, on the (right) side with distance correlated shadow fading switched on in the channel model of the multi-cell environment.

TABLE I

SySTEM LEVEL SimULATION ENVIRONMENT

\begin{tabular}{l|l} 
Parameter & Value \\
\hline Number of Cells & 19 sites / 3 sectors per site \\
Inter-site distance & $500 \mathrm{~m}$ \\
Carrier Frequency & $2.6 \mathrm{GHz}$ \\
UE Noise Figure & $9 \mathrm{~dB}$ \\
Pathloss & $17.75+37.6000 * \log 10$ (distance) \\
eNB parameters: & \\
Transmit Power & $46 \mathrm{dBm}$ \\
Antenna Model & $3 \mathrm{D} \mathrm{Antenna} \mathrm{Model} \mathrm{(Kathrein} \mathrm{80010541)} \mathrm{[6]}$ \\
Antenna Gain & $17 \mathrm{dBi}$ \\
Antenna Downtilt & $10^{\circ}$ \\
FWHM & $58^{\circ} \mathrm{azimuth}, 6.2^{\circ}$ elevation \\
Pico parameters: & \\
Transmit Power & $30 \mathrm{dBm}$ \\
Antenna Model & $3 \mathrm{D} \mathrm{Antenna} \mathrm{Model} \mathrm{(Kathrein} \mathrm{80010251)} \mathrm{[6]}$ \\
Antenna Gain & $11 \mathrm{dBi}$ \\
FWHM & $360^{\circ}$ azimuth, $7^{\circ}$ elevation \\
\hline
\end{tabular}

in [6]. Besides the common log-normal shadow fading model with standard deviation of $8 \mathrm{~dB}$, a geo-located correlation model with interand intra-site correlation of 0.5 and 1 respectively is applied, cf. [7], [8]. The auto-correlation distance is set to $50 \mathrm{~m}$. The LTE system is running in frequency division duplex (FDD) mode and using $20 \mathrm{MHz}$ bandwidth for both Macro and Pico cells. Standard channel models like extended spatial channel model (SCME) only model i.i.d shadow fading which is not very realistic in a real deployments. The SINR distribution of different HetNet scenarios with uncorrelated shadow fading and no shadow fading is shown in Fig. 2. HetNet deployments effect especially at the cell edge of Pico cells will require new RRM algorithm design. Shadowing channel effects will be taken into account on a system level basis. In order to benchmark practical RRM algorithms running in a time continuous mode, we apply a distancebased correlated shadow fading, shown in Fig. 1. By taking time windows and traffic models into accounts, these RRM algorithms can also model time delay, which is one KPI to be considered in the evaluations.

Results and Outlook: In the final paper we will evaluate the described RRM algorithms with the distributed precoding concept in a system level context using realistic antenna and shadow fading models, Fig. 2. Results will be evaluated according to standard KPIs

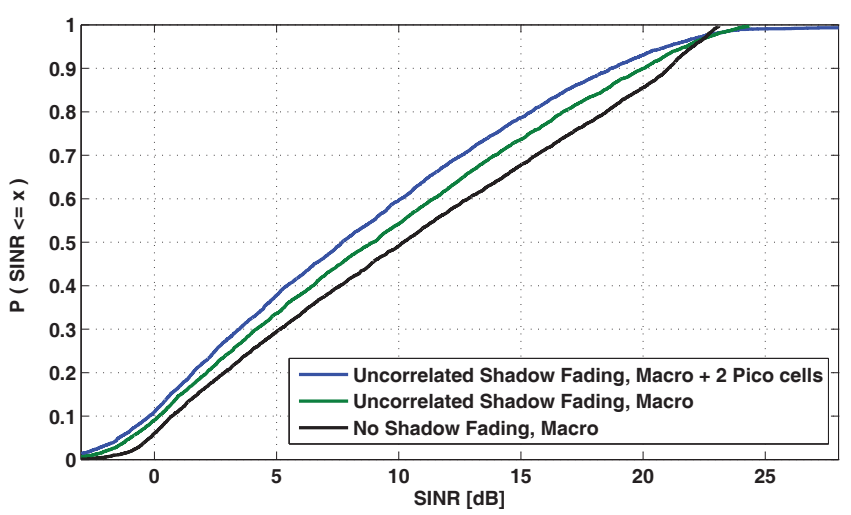

Fig. 2. System Level SINR distributions in HetNet scenarios: uncorrelated shadow fading model vs. no shadow fading

as well as new metrics with respect to the HetNet scenarios discussed. Under given RRM strategies, especially load on the X2 interface as well as power budget regarding Pico power consumption shall be taken into considerations.

\section{REFERENCES}

[1] Y. Sánchez, C. Hellge, T. Wirth, and T. Schierl, "Http live video streaming over mobile networks," IEEE COMSOC MMC E-Letter, vol. 6, no. 5, May 2011.

[2] Cisco White Paper, "Cisco visual networking index: Global mobile datatraffic forecast update, 2010 - 2015."

[3] S. Parkvall, E. Dahlman, A. Furuskar, Y. Jading, M. Olsson, S. Wanstedt, and K. Zangi, "Lte-advanced - evolving lte towards imt-advanced," in Vehicular Technology Conference, 2008. VTC 2008-Fall. IEEE 68th, sept. 2008 , pp. $1-5$.

[4] W. Mulder and T. Wirth, "Are we ready for the femtolution?" IEEE COMSOC MMC E-Letter, vol. 5, no. 5, Sep. 2010.

[5] 3GPP TR 36.814, "Further advancements for e-utra physical layer aspects (release 9)," March 2010.

[6] L. Thiele, T. Wirth, K. Brner, M. Olbrich, V. Jungnickel, J. Rumold, and S. Fritze, "Modeling of 3D Field Patterns of Downtilted Antennas and Their Impact on Cellular Systems," International ITG Workshop on Smart Antennas (WSA 2009), Feb. 2009.

[7] M. Gudmundson, "Correlation model for shadow fading in mobile radio systems," Electronics Letters, vol. 27, no. 23, pp. 2145 -2146, nov. 1991.

[8] H. Claussen, "Efficient modelling of channel maps with correlated shadow fading in mobile radio systems," in Personal, Indoor and Mobile Radio Communications, 2005. PIMRC 2005. IEEE 16th International Symposium on, vol. 1, sept. 2005, pp. $512-516$. 